\title{
The Formation of Carbon Nanostructures via Catalytic Pyrolysis of Naphthalene under Its Autogenic Pressure
}

\author{
Chao-Gang Wang, ${ }^{1}$ Xiao-Hong Shao, ${ }^{1}$ and Rui-Sheng Xue ${ }^{2}$ \\ ${ }^{1}$ College of Science, Beijing University of Chemical Technology, Beijing 100029, China \\ ${ }^{2}$ Key Laboratory of Carbon Fibers and Functional Polymers, Ministry of Education, Beijing University of Chemical Technology, \\ Beijing 100029, China
}

Correspondence should be addressed to Xiao-Hong Shao; shaoxh@mail.buct.edu.cn and Rui-Sheng Xue; xuersh@mail.buct.edu.cn Received 9 January 2015; Accepted 26 February 2015

Academic Editor: Yuhua Xue

Copyright (c) 2015 Chao-Gang Wang et al. This is an open access article distributed under the Creative Commons Attribution License, which permits unrestricted use, distribution, and reproduction in any medium, provided the original work is properly cited.

\begin{abstract}
The formation of carbon nanotubes (CNTs), spherical carbon nanocapsules (CNCs), and carbon spheres (CSs) is accomplished by using the method of reactions under autogenic pressure at elevated temperatures (RAPET). A powder mixture of naphthalene and nickel acetate tetrahydrate is dissociated under its autogenic pressure. The resultant CNTs and CNCs exhibit good graphitic quality, and the diameters range from $50 \sim 200 \mathrm{~nm}$. Smooth and monodisperse CSs with the diameter ranging from $5 \sim 10 \mu \mathrm{m}$ can be obtained by pyrolysis of pure naphthalene. Our results show that the reaction temperature and catalyst proportion play a key role in the formation of carbon nanostructures with RAPET method.
\end{abstract}

\section{Introduction}

Carbon nanotubes (CNTs), carbon nanocapsules (CNCs), and carbon spheres (CSs) are widely investigated due to their outstanding mechanical, electrochemical, and magnetic properties. The methods of syntheses for CNTs such as arc discharge, laser ablation, and chemical vapor deposition have broadly been examined. Meanwhile, various techniques have been applied to form the core-shell nanostructures, including the sol-gel method, carbon-arc techniques, and other techniques $[1,2]$.

The method of reactions under autogenic pressure at elevated temperatures (RAPET) is a quite simple, highly efficient, and economical method for its one-step reaction [3, 4]. Vilas prepared CSs by dissociating several hydrocarbons under their autogenic pressure; he found that the product morphology did not vary when the reaction time, amount of precursor, and reaction temperature were changed [5]. CNTs and core-shell nanostructures can be obtained by using the RAPET method to decompose carbon precursors at $700^{\circ} \mathrm{C}$ in the presence of several metal elements [6-9].

In this work, the utilization of naphthalene as an alternative carbon source and nickel acetate tetrahydrate as the catalyst for the synthesis of carbon materials by the RAPET method was experimentally investigated. The results indicate that the reaction temperature and catalyst proportion play a key role in the formation of carbon nanostructures.

\section{Experimental}

Naphthalene and nickel acetate tetrahydrate were all commercially available and used without further purification. For our syntheses, powder mixtures of naphthalene and nickel acetate tetrahydrate with different molar ratios $(3: 1,7: 1$, and $15: 1)$ were placed into an autoclave of $100 \mathrm{~mL}$ capacity at room temperature under air. Then, the filled autoclave was sealed tightly and placed inside a muffle furnace which was preheated to a certain temperature $(600,700,800,900$, or $1000^{\circ} \mathrm{C}$ ) for each sample. The precursor was then dissociated under its autogenic pressure. After 30 minutes, the reaction system was cooled to room temperature gradually, and the black product was obtained.

\section{Results and Discussion}

The morphology of the products was investigated by scanning electron microscope (SEM) and high-resolution transmission electron microscope (TEM). Figure 1 shows the SEM 


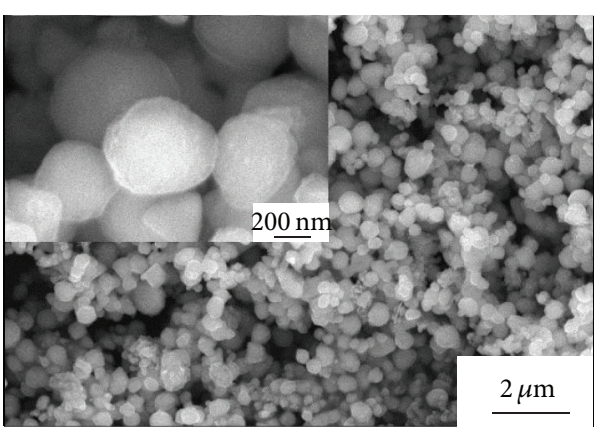

(a)

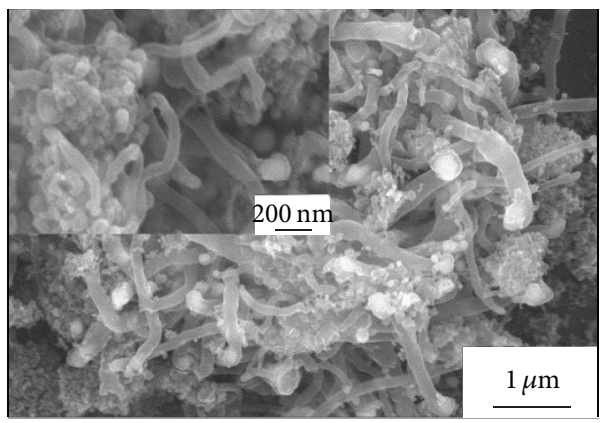

(c)

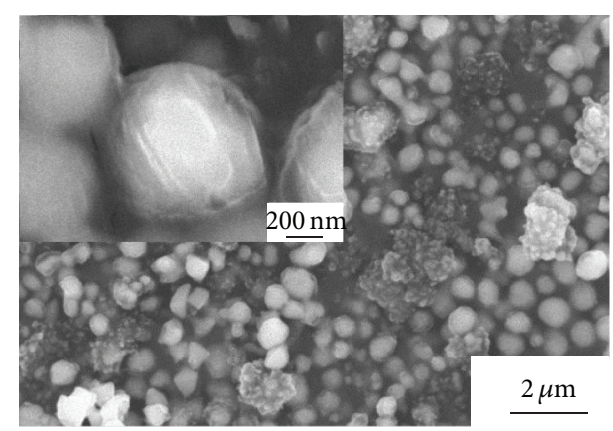

(b)

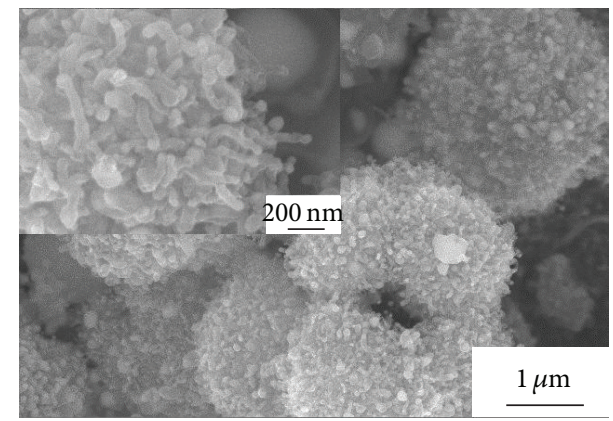

(d)

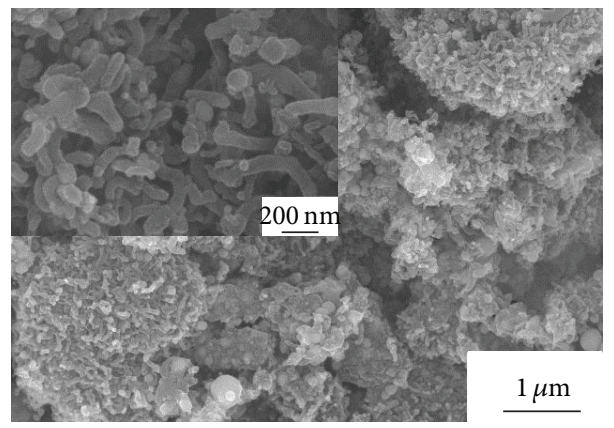

(e)

FIGURE 1: SEM images of products prepared from pyrolysis of $3.8 \mathrm{~g}$ naphthalene and $2.5 \mathrm{~g}$ nickel acetate tetrahydrate at different temperatures. The images of high magnification are in the left-top corner of (a)-(e), respectively. (a) $600^{\circ} \mathrm{C}$, (b) $700^{\circ} \mathrm{C}$, (c) $800^{\circ} \mathrm{C}$, (d) $900^{\circ} \mathrm{C}$, and (e) $1000^{\circ} \mathrm{C}$.

images of products which were fabricated from the mixture of naphthalene and nickel acetate tetrahydrate with the $3: 1$ molar ratio at different temperatures. From Figures 1(a) to $1(b)$, one can see that there are only spherical particles when the temperature is below $800^{\circ} \mathrm{C}$. Some synaptic structures appear on the surface of the products which have lost the spherical structure (Figure 1(b)), and we suggest that this is an initial stage of CNT forming process. One can see that the CNTs were obtained when the temperature reached $800^{\circ} \mathrm{C}$. With the increase of the temperature from $800^{\circ} \mathrm{C}$ to $1000^{\circ} \mathrm{C}$, the aspect ratio of CNTs is getting smaller and the diameters decrease from about $200 \mathrm{~nm}$ to $50 \mathrm{~nm}$, while the yield increases. It is also found that certain amounts of CNTs were tangled together to form a cluster. The result mentioned above is in reasonable agreement with those reported previously $[10,11]$. The increased temperature leads to a high-pressure reaction and a high diffusion rate of reactant mixture containing dissociated $\mathrm{Ni}$ nanoparticles and carbon atoms, which may facilitate the carbon atoms to dissolve in or deposit on the catalyst particle [10]. Meanwhile, the crystal water of nickel acetate tetrahydrate will be gasified at high temperature. The water vapor can be regarded as an activator due to its etching effect on amorphous carbon which was formed on the catalyst during pyrolysis. The increase of temperature will enhance the etching effect; therefore the deactivation of catalyst could slow down. Consequently, more CNTs with smaller nanometer size would be formed.

Figure 2 shows the SEM images of products, which were synthesized at $900^{\circ} \mathrm{C}$ with different molar ratios of naphthalene and nickel acetate tetrahydrate (pure naphthalene, $15: 1,7: 1$, and $3: 1$ ). Smooth and monodisperse CSs with diameters ranging from $5 \mu \mathrm{m}$ to $10 \mu \mathrm{m}$ can be obtained by pyrolysis of pure naphthalene (Figure $2(a)$ ). When the molar ratio of naphthalene and nickel acetate tetrahydrate is $15: 1$, the reaction products are composed of CSs and small particles (Figure 2(b)). The small particles are supposed to 


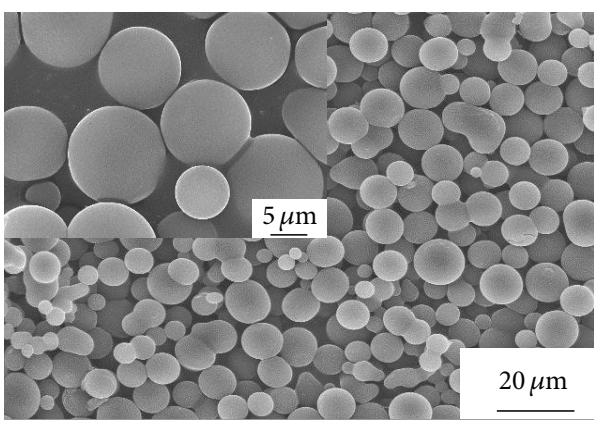

(a)

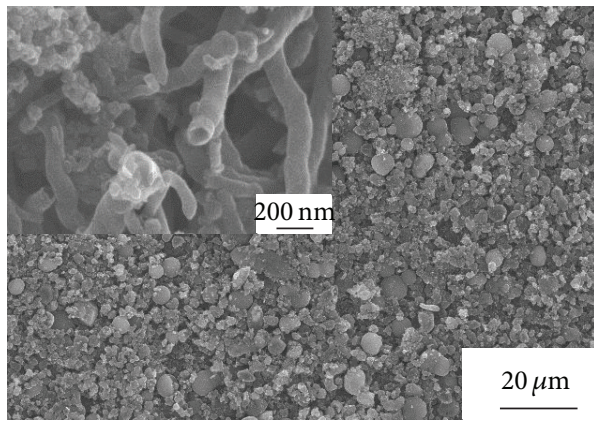

(c)

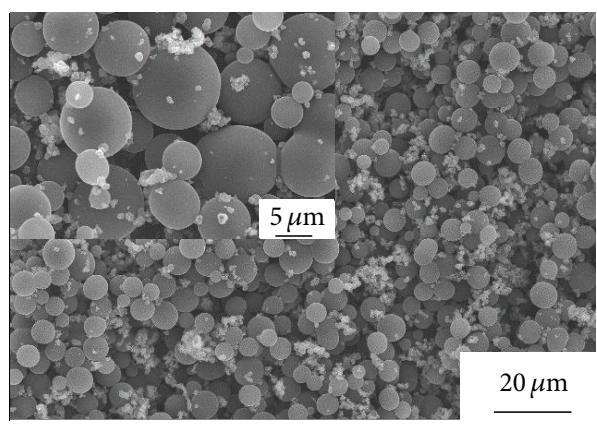

(b)

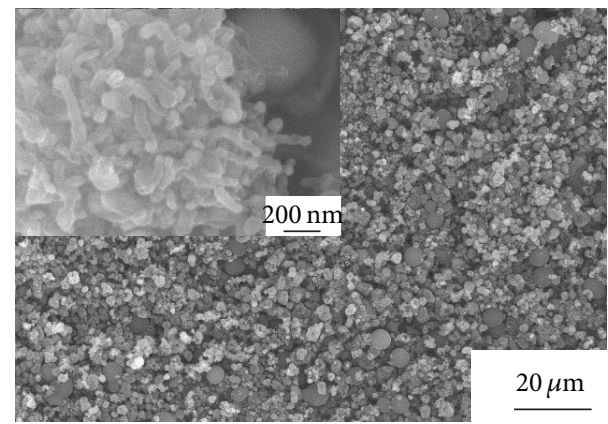

(d)

FIGURE 2: SEM images of products prepared at $900^{\circ} \mathrm{C}$ with different molar ratios of naphthalene/nickel acetate tetrahydrate. The images of high magnification are in the left-top corner of (a)-(d), respectively. (a) Pure naphthalene. (b), (c), and (d) are the images with the naphthalene/nickel acetate tetrahydrate ratios of $15: 1,7: 1$, and $3: 1$, respectively.

be produced from pyrolysis of the catalyst. Figure 2 also shows that the increase of the catalyst proportion leads to the decrease in the amount of CSs and the increase of CNTs and CNCs, which indicates that the catalyst is an essential factor for the formation and growth of CNTs and CNCs. Since the decomposition process of carbon source and catalyst is quite rapid in the closed RAPET system, the formation of different types of carbon species could occur in a short period of time. The carbon atoms disconnected with the catalysts could form CSs via self-assembly reaction, while the carbon atoms connected with the catalysts could form CNTs or CNCs. It is found that an increase of the catalyst proportion leads to the decrease in the amount of CSs and the increase of CNTs and CNCs, which indicates that the catalyst is an essential factor for the formation and growth of CNTs and CNCs.

Figure 3 is the XRD analysis of the samples produced with different molar ratios of naphthalene/nickel acetate tetrahydrate. Apparently, the sample of pure naphthalene only shows the diffraction peaks of carbon, while other samples reacted by different catalyst proportion show the diffraction peaks of both carbon and Ni. Furthermore, the structure becomes more regular with the increase of catalyst proportion.

TEM images (Figure 4) reveal clearly that there are $\mathrm{CNT}$ and spherical CNC nanostructures in the synthesized products. The spherical Ni nanoparticle is surrounded by graphitic shell to form the CNC structure, which may be formed by the continual growing of original surface shell around the $\mathrm{Ni}$ particle, and results in the overcoating and

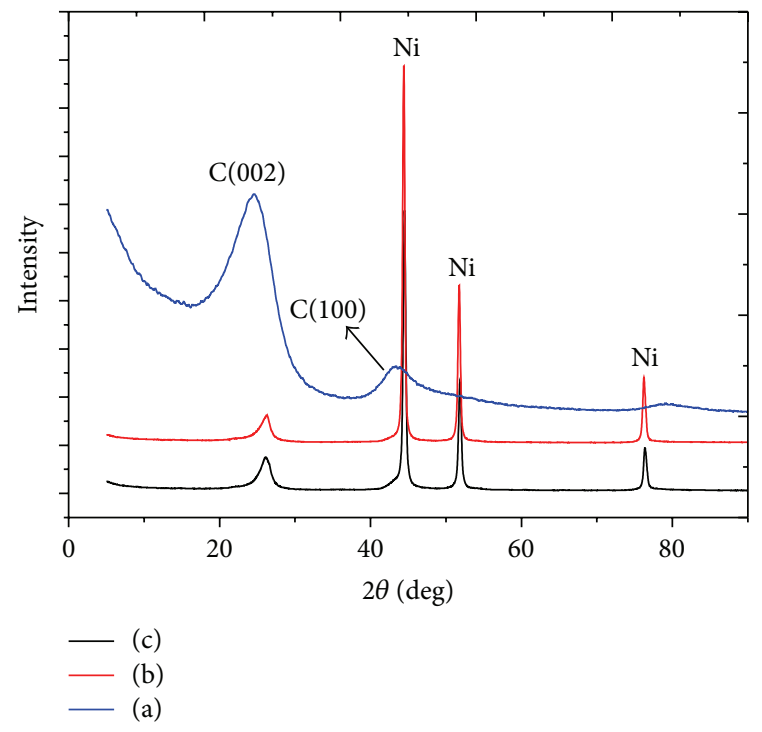

FIGURE 3: X-ray diffraction patterns of products prepared at $900^{\circ} \mathrm{C}$ with different molar ratios of naphthalene/nickel acetate tetrahydrate. (a) Pure naphthalene. (b) and (c) are the samples with the naphthalene/nickel acetate tetrahydrate ratios of $7: 1$ and $3: 1$, respectively.

deactivation of the catalyst (Figure 4(a)) $[12,13]$ ultimately. The insertion of pentagon or heptagon defects into the hexagonal graphite network can curl the resulting nanotubes 


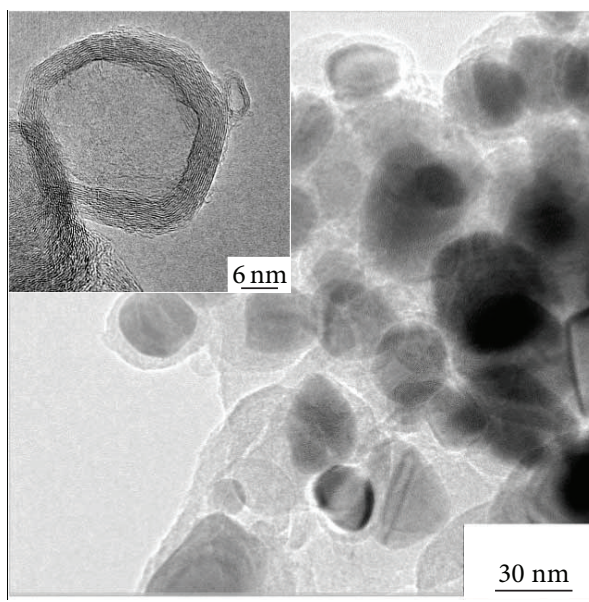

(a)

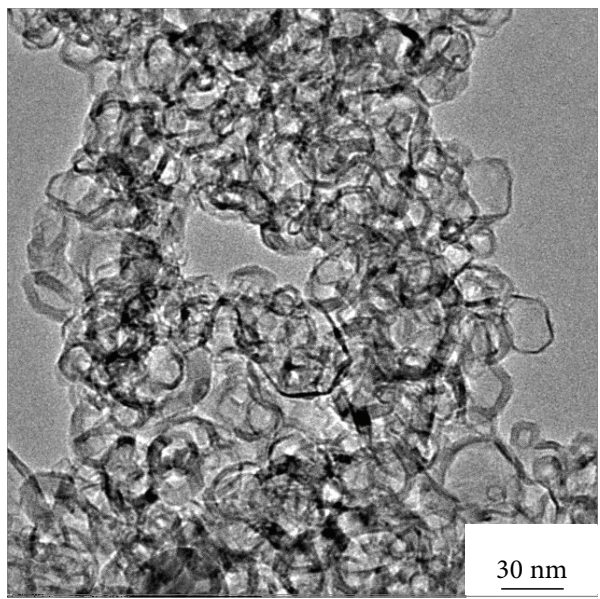

(c)

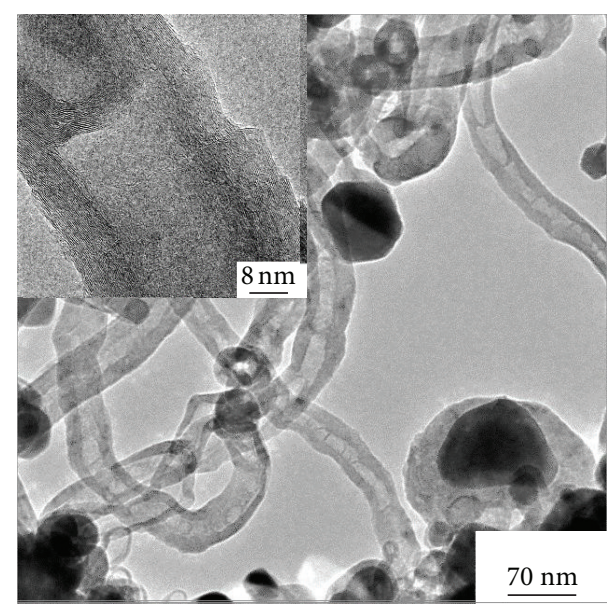

(b)

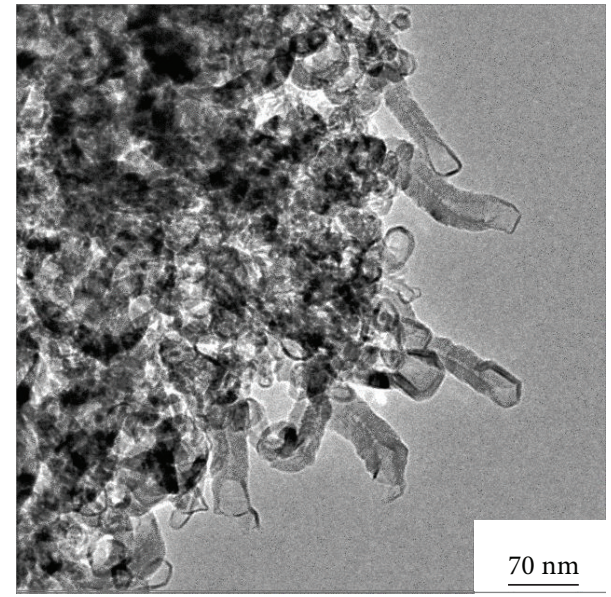

(d)

Figure 4: TEM images of CNCs and CNTs nanostructures prepared at $900^{\circ} \mathrm{C}$. The images of high magnification are in the left-top corner of (a)-(b), respectively. (a) and (b) are the images of CNCs and CNTs with the naphthalene/nickel acetate tetrahydrate ratio of $3: 1$. (c) and (d) are the images of CNCs and CNTs with the naphthalene/nickel acetate tetrahydrate ratio of $7: 1$ (after graphitization at $2800^{\circ} \mathrm{C}$ ).

[14]; the curving structure can then be formed. Also, it is possible that some carbon atoms could diffuse on the surface of the Ni particle and penetrate into the tube, forming a bridge structure across the nanotube wall. Therefore, the bamboolike structure could be formed inside a continuously growing nanotube (Figure 4(b)) [14]. Figures 4(c) and 4(d) reveal that the hollow CSs with regular graphite layers can be achieved by graphitization at $2800^{\circ} \mathrm{C}$ (Ar atmosphere: $-0.05 \mathrm{MPa}$ ). Obviously, the Ni particles were already removed during the graphitization process. The tangled CNTs are mixed with CNCs, indicating that the carbon shells could deactivate the catalysts. Meanwhile, CNT morphology was developed and induced only by the active catalyst particles.

\section{Conclusions}

In conclusion, CNTs and spherical CNCs were synthesized from pyrolysis of a mixture of naphthalene and nickel acetate tetrahydrate by using the RAPET method. Smooth and monodisperse CSs with diameters ranging from $5 \mu \mathrm{m}$ to
$10 \mu \mathrm{m}$ can be obtained by pyrolysis of pure naphthalene. Our results show that more CNTs and CNCs with smaller nanometer size will be formed with the increase of temperature in this closed RAPET system. In addition, the increase of the catalyst proportion leads to the decrease of the amount of CSs and the increase of CNTs and CNCs. The extra properties and potential application of this product will be addressed in our future work.

\section{Conflict of Interests}

The authors declare that there is no conflict of interests regarding the publication of this paper.

\section{Acknowledgments}

This work was supported by the National Nature Science Foundation of China under Grant no. 51102009. The authors are grateful to Professor Wenchuan Wang for his helpful discussion. 


\section{References}

[1] V. O. Nyamori, S. D. Mhlanga, and N. J. Coville, "The use of organometallic transition metal complexes in the synthesis of shaped carbon nanomaterials," Journal of Organometallic Chemistry, vol. 693, no. 13, pp. 2205-2222, 2008.

[2] J. Prasek, J. Drbohlavova, J. Chomoucka et al., "Methods for carbon nanotubes synthesis-review," Journal of Materials Chemistry, vol. 21, no. 40, pp. 15872-15884, 2011.

[3] S. V. Pol, V. G. Pol, and A. Gedanken, "Reactions under autogenic pressure at elevated temperature (RAPET) of various alkoxides: formation of metals/metal oxides-carbon core-shell structures," Chemistry - A European Journal, vol. 10, no. 18, pp. 4467-4473, 2004.

[4] H. Gershi, A. Gedanken, H. Keppner, and H. Cohen, “One-step synthesis of prolate spheroidal-shaped carbon produced by the thermolysis of octene under its autogenic pressure," Carbon, vol. 49, no. 4, pp. 1067-1074, 2011.

[5] V. G. Pol, S. V. Pol, J. M. Calderon Moreno, and A. Gedanken, "High yield one-step synthesis of carbon spheres produced by dissociating individual hydrocarbons at their autogenic pressure at low temperatures," Carbon, vol. 44, no. 15, pp. 32853292, 2006.

[6] V. G. Pol and P. Thiyagarajan, "Remediating plastic waste into carbon nanotubes," Journal of Environmental Monitoring, vol. 12, no. 2, pp. 455-459, 2010.

[7] V. G. Pol and M. M. Thackeray, "Spherical carbon particles and carbon nanotubes prepared by autogenic reactions: evaluation as anodes in lithium electrochemical cells," Energy and Environmental Science, vol. 4, no. 5, pp. 1904-1912, 2011.

[8] S. V. Pol, V. G. Pol, A. Frydman, G. N. Churilov, and A. Gedanken, "Fabrication and magnetic properties of ni nanospheres encapsulated in a fullerene-like carbon," Journal of Physical Chemistry B, vol. 109, no. 19, pp. 9495-9498, 2005.

[9] E. Holodelshikov, I. Perelshtein, and A. Gedanken, "Synthesis of air stable $\mathrm{FeCo} / \mathrm{C}$ alloy nanoparticles by decomposing a mixture of the corresponding metal-acetyl acetonates under their autogenic pressure," Inorganic Chemistry, vol. 50, no. 4, pp. 1288-1294, 2011.

[10] T. Charinpanitkul, N. Sano, P. Puengjinda, J. Klanwan, N. Akrapattangkul, and W. Tanthapanichakoon, "Naphthalene as an alternative carbon source for pyrolytic synthesis of carbon nanostructures," Journal of Analytical and Applied Pyrolysis, vol. 86, no. 2, pp. 386-390, 2009.

[11] Q. Li, H. Yan, J. Zhang, and Z. Liu, "Effect of hydrocarbons precursors on the formation of carbon nanotubes in chemical vapor deposition," Carbon, vol. 42, no. 4, pp. 829-835, 2004.

[12] H. Dai, A. G. Rinzler, P. Nikolaev, A. Thess, D. T. Colbert, and R. E. Smalley, "Single-wall nanotubes produced by metalcatalyzed disproportionation of carbon monoxide," Chemical Physics Letters, vol. 260, no. 3-4, pp. 471-475, 1996.

[13] R. T. K. Baker and P. S. Harris, "The formation of filamentous carbon," in Chemistry and Physics of Carbon, vol. 14, pp. 83-165, 1978.

[14] D. C. Li, L. M. Dai, S. M. Huang, A. W. H. Mau, and Z. L. Wang, "Structure and growth of aligned carbon nanotube films by pyrolysis," Chemical Physics Letters, vol. 316, no. 5-6, pp. 349$355,2000$. 

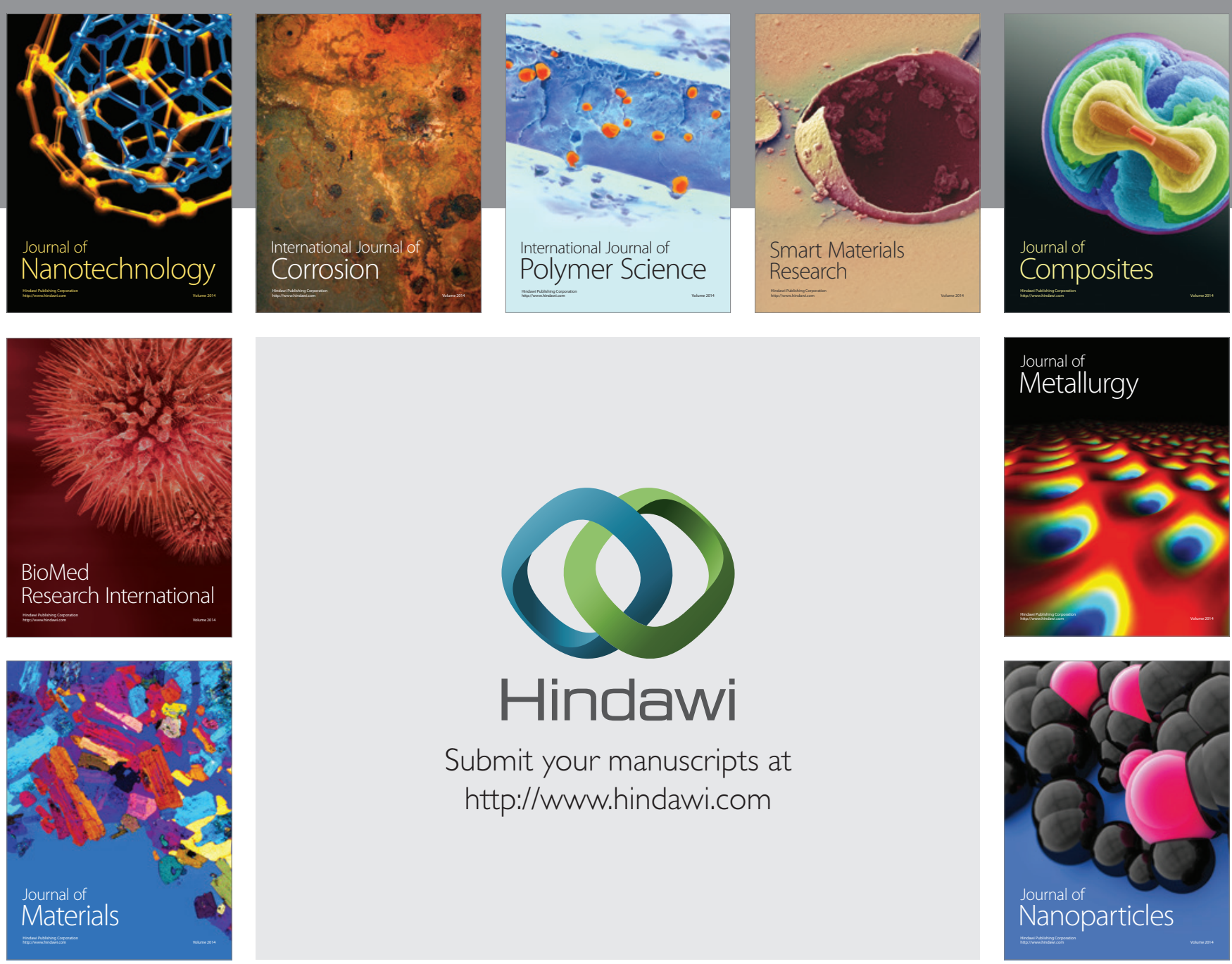

Submit your manuscripts at http://www.hindawi.com
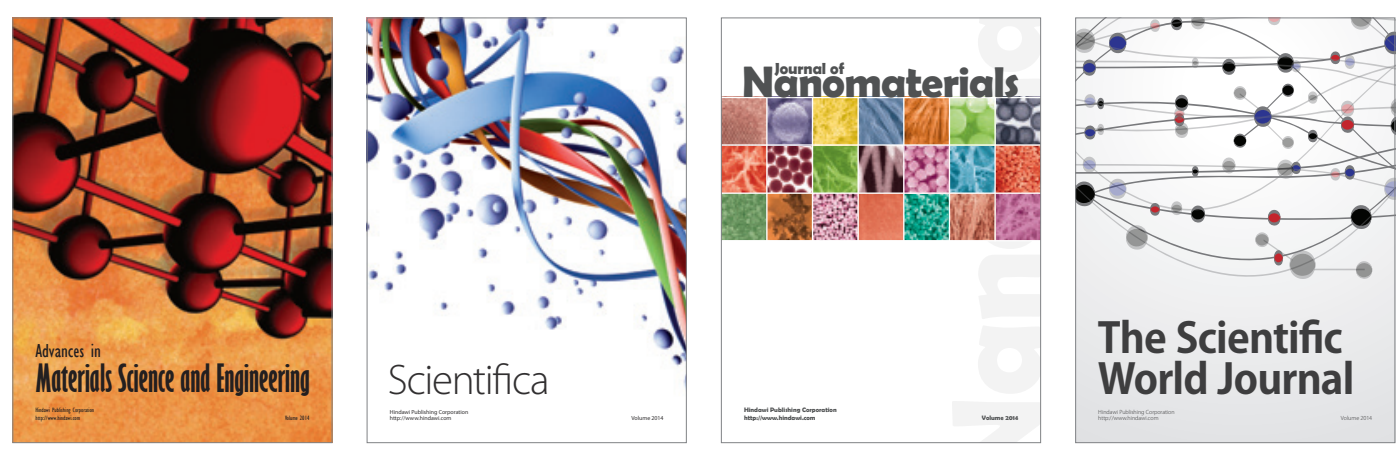

\section{The Scientific World Journal}
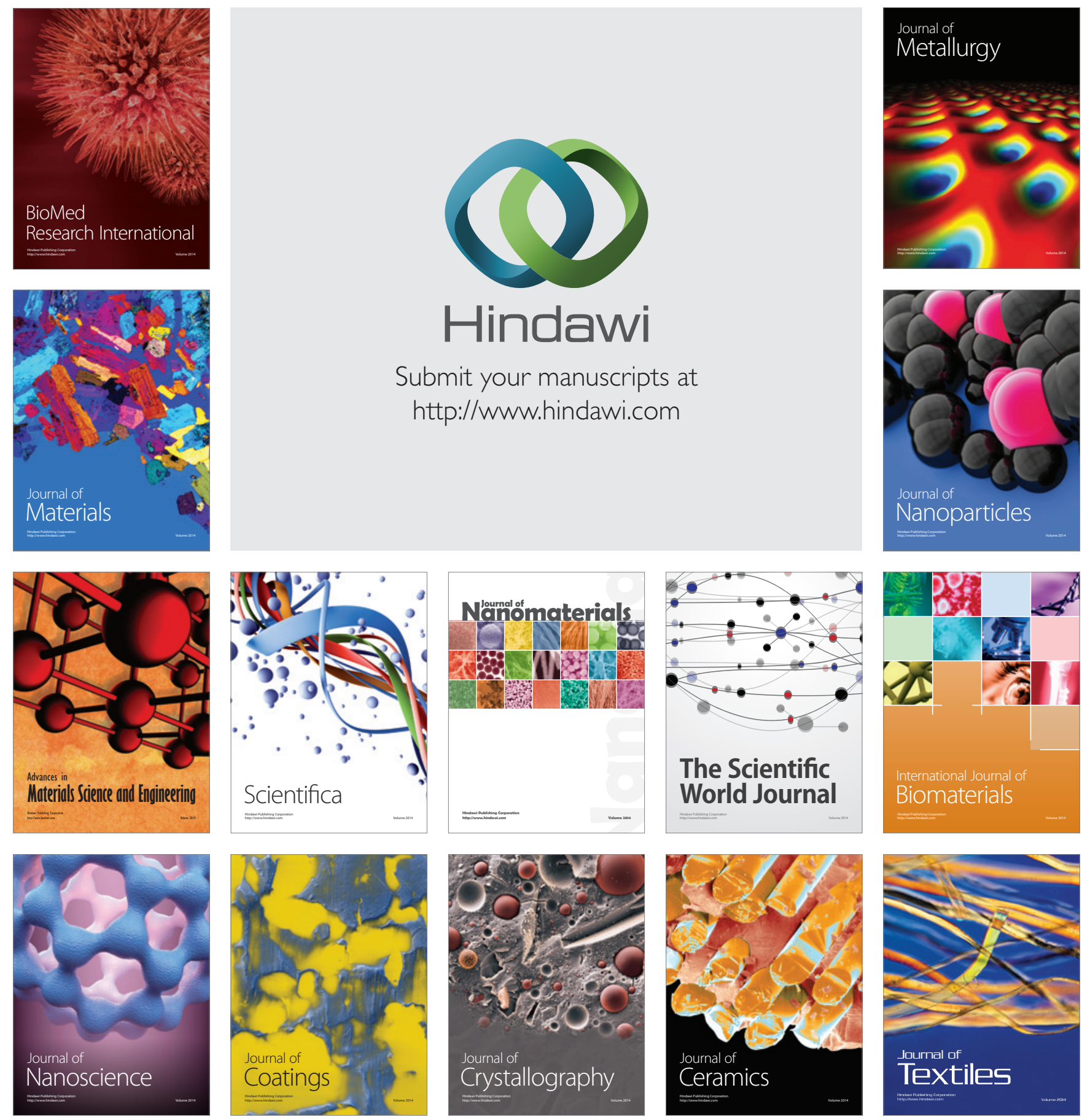